\title{
Focus in Honor of James A. McCloskey, Recipient of the 2005 ASMS Award for a Distinguished Contribution in Mass Spectrometry
}

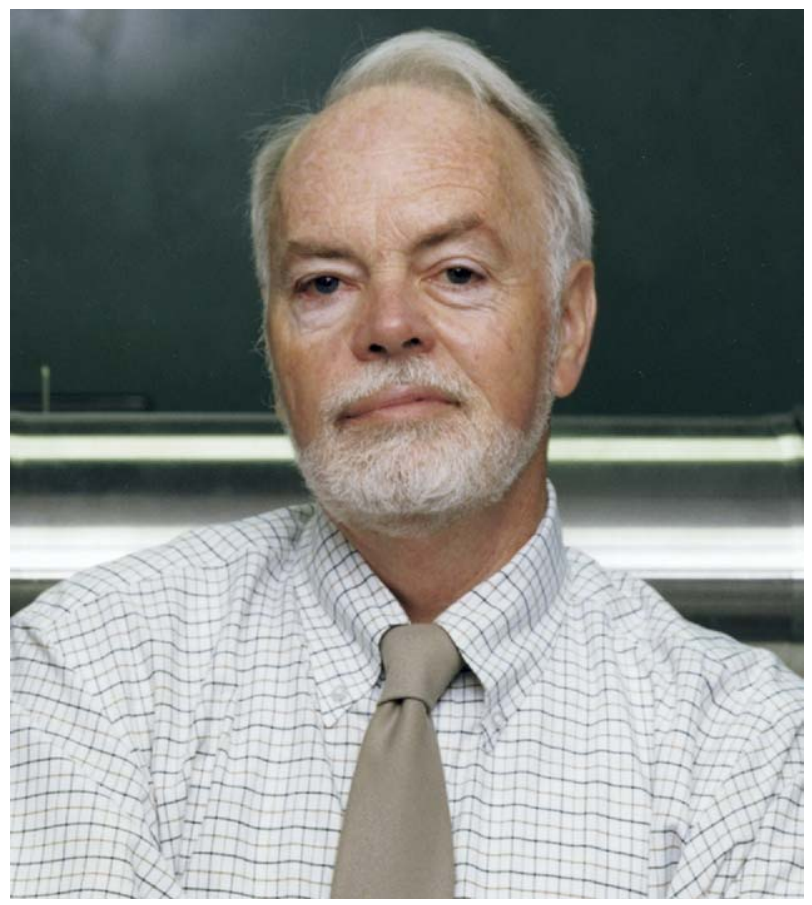

$\mathrm{I}$ $\mathrm{t}$ is rare to readily identify a single individual as the undisputed leader in the practice of mass spectrometry for a particular field of study. Even more rare is the individual who has maintained an unparalleled level of success in his or her chosen field for over 35 years. Professor Jim McCloskey is such an individual. Jim's singular achievement has been to pioneer and continually advance the frontiers of mass spectrometry in the analysis of nucleic acid constituents. For his accomplishments, he received the 2005 ASMS Award for Distinguished Contribution in Mass Spectrometry at the 53rd ASMS Conference in his hometown of San Antonio, Texas.

Jim was born in San Antonio and received his B.S. from Trinity University in 1957. He then went on to MIT to work with a young assistant professor who had recently begun his career there, Klaus Biemann, receiving his Ph.D. in 1963. During that period, Jim also spent several years in the US Army. His work in the field of

Published online July 27, 2006 nucleic acid constituents can be traced back to his years in the Biemann lab with one of his very first publications being a Journal of the American Chemical Society (JACS) communication on the EI spectra of nucleosides. Jim spent two years as a post-doc in France (developing an abiding affinity for that country) and then came back to Baylor College of Medicine to begin his academic career. He quickly progressed through the tenure and promotion process, eventually moving to his current position in the Department of Medicinal Chemistry at the University of Utah in 1974. Jim formally retired as an active faculty member in 2005.

Jim has contributed over 250 publications to the literature in his field, and his work impacts significantly on numerous areas of science including chemistry, biochemistry, molecular and cell biology, and pharmacology. Applications of mass spectrometry to nucleic acid constituents have historically been challenging owing to the high intrinsic polarity of nucleosides and (oligo)nucleotides and the problems associated with their conversion into gaseous ions. Jim's independent 
contribution can be traced as far back as 1968 when he published the seminal work on derivatization of nucleic acid components for their analysis by GC-MS. This accomplishment was crucial in extending the use of mass spectrometry into the area of nucleic acids. As a result, mass spectrometry has played a dominant role in the structure determination of new nucleosides from nucleic acids and other sources. Of the more than 100 known nucleosides in RNA, nearly all of those discovered since Jim's seminal paper have exclusively relied on mass spectrometry as the method of structural elucidation

As instrumentation and techniques in mass spectrometry evolved in the 1980's and 1990's, Jim was quick to seize upon these new capabilities to extend mass spectrometry further into the structural characterization of nucleic acids. His lab developed and applied LC-MS and ESI-MS/MS approaches to nucleoside/ nucleotide structural characterization, and these approaches are now common tools used by biochemists and structural biologists who require structural identification of nucleic acid constituents. Moreover, these approaches form the core of Jim's efforts, originally initiated in the 1970's and continuing to this day, in using mass spectrometric techniques for structurally identifying modified nucleosides in a variety of RNAs with the aim to couple the studies of the chemistry of RNA modifications with the underlying biology.

Jim's accomplishments in the field of mass spectrometry are simply outstanding and also quite significant in the field of biological chemistry as he almost singlehandedly demonstrated the unique power of using sophisticated techniques in mass spectrometry to study modified bases critically involved in the function of various RNA molecules. He has not wavered from this singular focus, and has continually applied the most recent techniques in mass spectrometry to his biochemical studies. As a result, mass spectrometry is the premier technique in this area of biomedical research. In no small way, this has advanced mass spectrometry by revealing its utility to unravel mysteries of nucleic acid biology in the broadest sense. His focused and dedicated research approach has served as a role model for many investigators by showing that a targeted research program in applied mass spectrometry can have enormous payoffs.

This Focus issue highlights activities by the next generation of mass spectrometrists interested in nucleic acids. The first group of contributed articles discuss continuing efforts to understand the fundamentals of ribose, nucleoside and oligonucleotide reactivity and fragmentation. Hilkka Kenttamaa and coworkers begin the Focus with a report on the reactivity of riboses. Huachuan Cao with Yinsheng Wang discuss the collisionally activated dissociation (CAD) pathways of modified cytidines; these fragmentations have implications in assay development for detecting DNA damage products. In a related vein, Jennifer Brodbelt and coworkers use ESI-MS and CAD approaches to study cytosine adducts. Finn Kirpekar and coworkers and Kristina Hakånsson and Jiong Yang discuss the fragmentation of oligoribonucleotides by CAD and ion-electron methods, respectively. In the second group of contributed articles, the use of mass spectrometry to understand nucleic acid structure is illustrated. Jason Kieltyka and Christine Chow use inorganic complexes to probe RNA hairpin structures with a particular emphasis on illustrating how ESI-MS is appropriate for studying metalRNA binding complexes. Next, Jeehiun Lee and coworkers investigate correlations between DNA duplex behavior in the gas phase versus that observed in solution. The final group of articles demonstrate the roles modern mass spectrometry can play in understanding the chemistry and biology of nucleic acids. Arthur Van Aerschot and Jef Rozenski illustrate the important role of mass spectrometry in development of thiolated deoxyoligonucleotides used in microarray construction. Finally, Dan Fabris and coworkers examine the aminogylcoside binding domains of the HIV-1 packaging signal RNA using tandem mass spectrometry.

Without question, each of these contributors has been influenced by Jim's seminal work in the field, and all continue to build upon his pioneering efforts. Those of us who have had the pleasure of knowing and working with Jim personally can testify to his infectious enthusiasm for science, his appreciation for the inherent beauty of nature, and his warm and engaging interactions with everyone he meets. While Jim is a humble man by nature, it is a distinct and sincere honor to recognize his accomplishments and contributions in this issue of JASMS. Congratulations, Jim!

Pat Limbach, Guest Editor Dave Muddiman, Guest Editor 